\title{
EXTENSION D'UN THÉORÈME DE LOUIS ANTOINE
}

\author{
NIKIAS STAVROULAKIS
}

Abstract. Let $f:[0,1] \times S \rightarrow R^{3}$ be a map subject to the conditions: (1) $f \mid] 0,1] \times S$ is $(1,1)$ into; (2) $f \mid\{0\} \times S$ is not $(1,1)$ into; (3) The image $\Gamma_{0}=f(\{0\} \times S)$ is a Jordan curve; (4) $f(\{0\} \times S) \cap$ $f(10,1] \times S)=\varnothing$.

Let $\mu$ be the linking number of each of the curves $\Gamma_{t}=f(\{t\} \times S)$, $t \in] 0,1]$, with $\Gamma_{0}$. Let $v$ be the degree of the mapping $h: S \rightarrow \Gamma_{0}$ defined by $h(u)=f(0, u)$. We prove that, if $\Gamma_{0}$ is tame, the integers $\mu$ and $v$ are relatively prime. The question is open in case that $\Gamma_{0}$ is wild.

1. Position du problème. Soit $S$ le quotient de $R$ par la relation d'équivalence $u \equiv v(\bmod 1)$. Sur un tore plongé dans $R^{3}$ moyennant les équations

$$
\begin{aligned}
& x_{1}=\left(a_{1}+a \cos 2 \pi \varphi_{1}\right) \cos 2 \pi \varphi_{2}, \\
& x_{2}=\left(a_{1}+a \cos 2 \pi \varphi_{1}\right) \sin 2 \pi \varphi_{2}, \\
& x_{3}=a \sin 2 \pi \varphi_{1} \quad\left(a_{1}>a \geqq 1, \varphi_{1} \in S, \varphi_{2} \in S\right),
\end{aligned}
$$

on considère une courbe de Jordan $\Gamma$ ainsi que ses nombres d'enlacement $\mu$ et $\nu$, considérés non négatifs, avec $\Gamma_{0}:\left(x_{1}^{2}+x_{2}^{2}=a_{1}^{2}, x_{3}=0\right)$ et avec l'axe des $x_{3}$. L. Antoine a démontré [1] que, si $\mu>1$ et $v>1$, ces deux entiers sont premiers entre eux. Ce théorème exprime une propriété indépendante du plongement, parce que $\mu$ et $v$ sont respectivement les degrés des projections canoniques de $\Gamma$ dans un méridien et dans un parallèle du tore. Mais la considération du plongement conduit à un nouveau problème. En effet, $\Gamma$ est définie par des fonctions continues $\varphi_{1}(u)$ et $\varphi_{2}(u)$ de $u \in S$, donc remplaçant dans (1) et faisant varier $a=t$ sur $[0,1]$, on obtient une application continue $g:[0,1] \times S \rightarrow R^{3}$ telle que $\left.\left.g \mid\right] 0,1\right] \times S$ soit injective et que, d'une part $\mu$ soit le nombre d'enlacement de toute courbe $g(\{t\} \times S)$, $t \in] 0,1]$, avec $\Gamma_{0}$, d'autre part $v$ soit le degré de l'application $\xi: S \rightarrow \Gamma_{0}$ définie par $\xi(u)=g(0, u)$. Les entiers $\mu$ et $\nu$ se déterminent ainsi par l'application $g$ qui donne lieu à une figure relativement simple dans le cas envisagé. Il est donc naturel de chercher à généraliser la situation en introduisant partout des figures sauvages de $R^{3}$ comme suit.

Received by the editors May 19, 1972.

AMS (MOS) subject classifications (1970). Primary 55A25, 55A30; Secondary 54F20, 55A20.

(c) American Mathematical Society 1973 
Soit $f:[0,1] \times S \rightarrow R^{3}$ une application continue jouissant des propriétés suivantes: (1) La restriction de $f$ à $] 0,1] \times S$ est injective. (2) La restriction de $f$ à $\{0\} \times S$ n'est pas injective mais l'image $\Gamma_{0}=f(\{0\} \times S)$ est une courbe de Jordan dans $R^{3}$. (3) $\left.\left.f(\{0\} \times S) \cap f(] 0,1\right] \times S\right)=\varnothing$. Toutes les courbes de Jordan $\left.\left.\Gamma_{t}=f(\{t\} \times S), t \in\right] 0,1\right]$, ont alors avec $\Gamma_{0}$ un même nombre d'enlacement qui sera noté $\mu$. Soit $v$ le degré de l'application $h: S \rightarrow \Gamma_{0}$ définie par $h(u)=f(0, u)$.

Question. Si $\mu>1$ et $v>1$, est-ce que ces deux entiers sont premiers entre eux?

Si $f$ est telle que la réponse soit affirmative, on obtient une généralisation du théorème d'Antoine. Le Corollaire 3.3 dit qu'il en est ainsi lorsque $\Gamma_{0}$ est une ligne polygonale, donc aussi lorsque $\Gamma_{0}$ est non sauvage. La Proposition 3.3 concerne le cas général. Les Propositions 3.1 et 3.2 peuvent aussi être considérées comme généralisations du théorème d'Antoine.

2. Propositions auxiliaires. Par cycle polygonal nous entendons une courbe de Jordan formée d'un nombre fini de segments rectilignes. Par contre un 1-cycle; considéré comme chaîne, ne sera pas dépourvu en général de points multiples. Deux courbes de Jordan disjointes dans $R^{3}$ seront dites simplement enlacées si leur nombre d'enlacement est 1.

Proposition 2.1. f étant définie comme précédemment, soit L un cycle polygonal simplement enlacé avec $\Gamma_{0}$, ce qui entraîne que toutes les courbes $\Gamma_{t}$ pour $0<t \leqq \varepsilon \leqq 1, \varepsilon$ convenable, ont un même nombre d'enlacement avec L. Ce nombre est égal à $\nu$.

DÉmonstration. Soient $\theta>0$ suffisamment petit et $z_{1}=\sum\left(a_{i} a_{i+1}\right)$ un 1-cycle situé dans un $\theta$-voisinage de $\Gamma_{0}$ et défini par des points $a_{1}, a_{2}, \cdots$, $a_{n}$ de $\Gamma_{0}$ pris dans un ordre cyclique. Soit $z_{2}=\sum s_{k}$ une 2-chaîne ayant pour bord $L$ et en position canonique relativement à $z_{1}$. Pour chaque 2-simplexe $s_{k}$ de $z_{2}$ ayant une intersection non vide avec $z_{1}$, on considère les 1simplexes $l_{k_{i}}=a_{k_{i}} a_{k_{i}+1}$ de $z_{1}$ rencontrant $s_{k}$, ce qui donne $s_{k} \otimes l_{k_{i}}=\varepsilon_{k_{i}}= \pm 1$ et $\sum_{k} \sum_{i} \varepsilon_{k_{i}}=1$, le signe du nombre d'intersection $\varepsilon_{k_{i}}$ dépendant des orientations respectives des $s_{k}$ et $l_{k_{i}}$.

Le paramètre $r$ parcourant une copie $S^{\prime}$ de $S$, il existe une injection continue $S^{\prime} \rightarrow R^{3}$ ayant pour image la courbe $\Gamma_{0}$. Par conséquent $h$ induit une application continue $\varphi: S \rightarrow S^{\prime}$ telle que $r=\varphi(u)= \pm v u+\psi(u), \psi(u)$ étant périodique de période 1 . Changeant au besoin le signe de $r$, on a toujours $r=v u+\psi(u)$.

Soient $r_{1}, r_{2}, \cdots, r_{n}$ les valeurs de $r$ définissant les points $a_{1}, a_{2}, \cdots, a_{n}$, et choisissons, pour chaque $r_{i}$, une valeur $u_{i}^{\prime}$ de $u$ telle que $\varphi\left(u_{i}^{\prime}\right)=r_{i}$. Les points $u_{i}^{\prime} \in S$, pris dans l'ordre cyclique convenable, définissent un 1cycle qui peut être subdivisé de façon que, sur le 1-cycle qui en résulte 
$\left(u_{1} u_{2} \cdots u_{p} u_{1}\right), \varphi$ admette une approximation simpliciale $\varphi_{\sigma}$. Les restrictions des $\varphi$ et $\varphi_{\sigma}$ à l'ensemble des sommets $u_{j}$ sont identiques.

Prenant un réel positif $t<\varepsilon$ et les points $b_{j}=f\left(t, u_{j}\right)(j=1,2, \cdots, p)$ de $\Gamma_{t}$, on obtient un 1-cycle $z_{1 t}=\sum\left(b_{j} b_{j+1}\right)$ dans $R^{3}$ et une application simpliciale $\varphi_{\sigma t}$, induite par $\varphi_{\sigma}$, de $z_{1 t}$ sur $z_{1}$. Si $\varphi_{\sigma}\left(u_{j}\right)=r_{i}$, on a $\varphi_{\sigma t}\left(b_{j}\right)=a_{i}=$ $f\left(0, u_{j}\right)$, donc les distances $\left|b_{j}-a_{i}\right|$ tendent uniformément vers zéro avec $t$. Par conséquent il existe un réel positif $t<\varepsilon$ donnant lieu aux propriétés suivantes: (1) Le cycle $z_{1 t}$ ne rencontre pas $L$. (2) Un 1-simplexe fermé de $z_{1 t}$ qui ne s'applique pas par $\varphi_{\sigma t}$ sur un 1-simplexe $l_{k_{i}}$ ne rencontre pas le support fermé de $z_{2}=\sum s_{k}$. (3) Si un 1-simplexe $l_{k_{i j}}$ de $z_{1 t}$ s'applique par $\varphi_{\sigma t}$ sur un simplexe $l_{k_{i}}$ de $z_{1}$, les intérieurs des $l_{k_{i j}}$ et $s_{k}$ ont un seul point commun. Soit $m_{k_{i}}$ (resp. $m_{k_{i}}^{\prime}$ ) le nombre des simplexes $l_{k_{i j}}$ qui sont orientés comme $l_{k_{i}}\left(\right.$ resp. comme $-l_{k_{i}}$ ) par rapport à $s_{k}$. Comme $m_{k_{i}}-m_{k_{i}}^{\prime}=v$, le nombre d'enlacement de $z_{1 t}$ avec $L$ est $\sum_{k} \sum_{i}\left(m_{k_{i}}-m_{k_{i}}^{\prime}\right) \varepsilon_{k_{i}}=v$.

DéFINITION. On appelle surface simpliciale de $R^{3}$ un polyèdre triangulé de $R^{3}$ qui est l'image d'une surface compacte connexe, avec ou sans bord, par une injection continue. Les 2 -simplexes (faces triangulaires) de ce polyèdre seront toujours considérés fermés.

Définition. Deux surfaces simpliciales $A_{1}$ et $A_{2}$ de $R^{3}$ se trouvent en position relative canonique si (1) aucun sommet de $A_{1}$ (resp. de $A_{2}$ ) n'appartient à $A_{2}$ (resp. à $A_{1}$ ), (2) aucune face de $A_{1}$ (resp. de $A_{2}$ ) ne contient un segment appartenant à une arête de $A_{2}$ (resp. de $A_{1}$ ), (3) l'intérieur d'une arête quelconque de $A_{1}$ ne rencontre l'intérieur d'aucune arête de $A_{2}$.

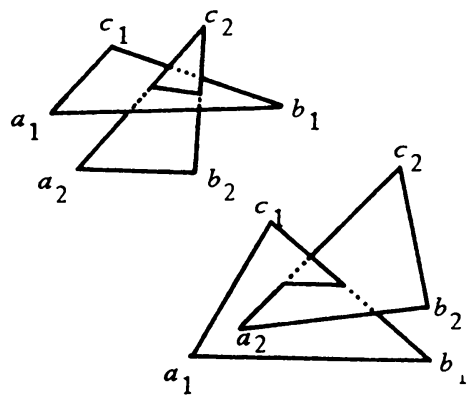

Les surfaces simpliciales $A_{1}$ et $A_{2}$ étant en position relative canonique, si la face $a_{1} b_{1} c_{1}$ de $A_{1}$ rencontre la face $a_{2} b_{2} c_{2}$ de $A_{2}$, alors les plans de ces deux faces sont distincts et l'intersection $\left(a_{1} b_{1} c_{1}\right) \cap\left(a_{2} b_{2} c_{2}\right)$ est un segment dont chacune des extrémités est intérieure à l'une des deux faces et aussi intérieure à une arête de l'autre face.

Proposition 2.2. Soient $A_{1}$ et $A_{2}$ deux surfaces simpliciales de $R^{3}$ en position relative canonique. Si l'intersection $A_{1} \cap A_{2}$ n'est pas vide et ne 
contient aucun point des bords des $A_{1}$ et $A_{2}$, elle est constituée par un nombre fini de cycles polygonaux deux à deux disjoints.

En effet, une composante connexe de $A_{1} \cap A_{2}$ est la réunion d'un nombre fini de segments $l_{1}, l_{2}, \cdots, l_{n}$ dont chacun est l'intersection d'une face de $A_{1}$ et d'une face de $A_{2}$. Si $l_{i} \cap l_{j} \neq \varnothing, l_{i} \cap l_{j}$ se réduit à un point qui est extrémité de chacun des segments $l_{i}$ et $l_{j}$, d'après la propriété signalée ci-dessus. La même propriété montre que si $a$ est extrémité d'un segment $l_{i}$, il sera aussi extrémité d'un autre segment unique $l_{j}$, d'où le résultat.

Lemme 2.1. Soient ab et $c d$ deux segments rectilignes de $R^{3}$ sans points communs. L'ensemble $E$ des points $e \in R^{3}$ pour lesquels les triangles fermés eab et ecd sont non dégénérés et ont pour intersection le sommet e, est un ouvert non vide de $R^{3}$.
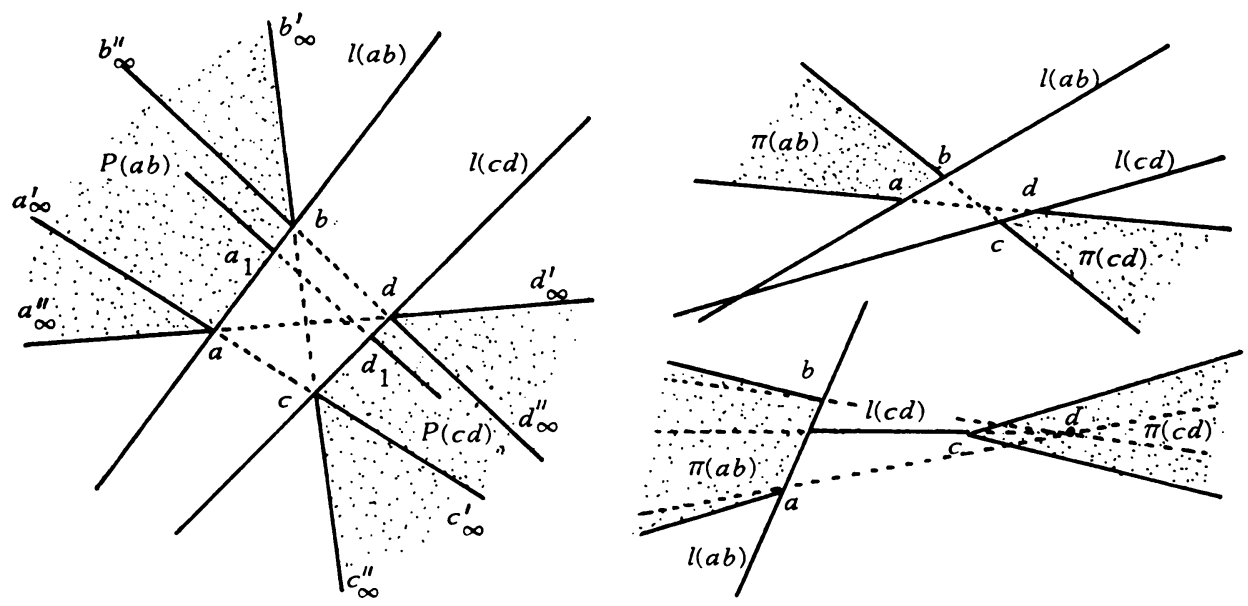

Démonstration. Soient $l(a b)$ et $l(c d)$ les droites définies par $a b$ et $c d$. Les triangles $e a b$ et $e c d$ étant non dégénérés, on a $l(a b) \subset R^{3}-E$ et $l(c d) \subset$ $R^{3}-E$.

Supposons premièrement que $a b$ et $c d$ soient non coplanaires. Si $e^{\prime} \in R^{3}-(l(a b) \cup l(c d))$ et si l'intersection $\left(e^{\prime} a b\right) \cap\left(e^{\prime} c d\right)$ ne se réduit pas au point $e^{\prime}$, elle sera un segment définissant une droite $l\left(a_{1} d_{1}\right)$ rencontrant $a b$ et $c d$. Soient $a_{1}=(a b) \cap l\left(a_{1} d_{1}\right)$ et $d_{1}=(c d) \cap l\left(a_{1} d_{1}\right)$. Comme $e^{\prime}$ ne peut pas être intérieur au segment $a_{1} d_{1}$, considérant les polyèdres fermés $P(a b)=$ $a_{\infty}^{\prime \prime} a_{\infty}^{\prime} a b b_{\infty}^{\prime} b_{\infty}^{\prime \prime}$ et $P(c d)=c_{\infty}^{\prime \prime} c_{\infty}^{\prime} c d d_{\infty}^{\prime} d_{\infty}^{\prime \prime}$, comme l'indique la figure, on voit que $E=R^{3}-(l(a b) \cup l(c d) \cup P(a b) \cup P(c d))$ est un ouvert non vide de $R^{3}$.

Supposant deuxièmement que $a b$ et $c d$ appartiennent à un plan $\pi$, on a $l(a b) \subset R^{3}-E, \quad l(c d) \subset R^{3}-E$, et aussi comme l'indiquent les figures, 
$\pi(a b) \subset R^{3}-E, \pi(c d) \subset R^{3}-E$, donc $E=R^{3}-(l(a b) \cup l(c d) \cup \pi(a b) \cup \pi(c d))$ est un ouvert non vide de $R^{3}$.

Proposition 2.3. Soient $A_{1}$ et $A_{2}$ deux surfaces simpliciales de $R^{3}$, $A_{2}$ étant sans bord, et supposons que $A_{1} \cap A_{2}$ soit non vide et ne contienne aucun point du bord $\partial A_{1}$. Moyennant une suite finie d'isomorphismes simpliciaux n'affectant pas $\partial A_{1}$, on peut transformer $A_{1}$ en une surface simpliciale isomorphe à $A_{1}$ et se trouvant en position relative canonique avec $A_{2}$.

Démonstration. Dans la suite $B_{a}(\theta)$ désigne une boule ouverte de centre $a$ et de rayon $\theta$.

(I) Supposons qu'un sommet $a$ de $A_{1}$ soit aussi un sommet de $A_{2}$. Soient, prises dans un ordre cyclique, $a a_{1} a_{2}, a a_{2} a_{3}, \cdots, a a_{m} a_{1}$ les faces de $A_{1}$ autour de $a$.

Soit $\varepsilon_{i}>0$ tel que, pour tout $a^{\prime} \in B_{a}\left(\varepsilon_{i}\right), a^{\prime} a_{i} a_{i+1}$ n'ait aucun point commun avec le complémentaire du disque fermé $\left(a a_{1} a_{2}\right) \cup\left(a a_{2} a_{3}\right) \cup \cdots \cup$ $\left(a a_{m} a_{1}\right)$ dans $A_{1}[i=1,2, \cdots, m ; m+1=1(\bmod m)]$.

Déterminons, moyennant le Lemme $2.1, \varepsilon^{\prime}>0$ tel que, pour tout $a^{\prime} \in B_{a}\left(\varepsilon^{\prime}\right)$, tous les triangles $a^{\prime} a_{i} a_{i+1}$ soient non dégénérés et que $\left(a^{\prime} a_{i} a_{i+1}\right) \cap\left(a^{\prime} a_{j} a_{j+1}\right)=a^{\prime}$ toutes les fois où les arêtes $a_{i} a_{i+1}$ et $a_{j} a_{j+1}$ n'ont pas d'extrémité commune.

Pour tout sommet $b_{k} \neq a$ de $A_{2}$ n'appartenant pas au disque $\left(a a_{1} a_{2}\right) \cup$ $\cdots \cup\left(a a_{m} a_{1}\right)$, soit $\theta_{k}>0$ tel que $b_{k} \notin\left(a^{\prime} a_{1} a_{2}\right) \cup \cdots \cup\left(a^{\prime} a_{m} a_{1}\right)$ quel que soit $a^{\prime} \in B_{a}\left(\theta_{k}\right)$.

Si $\varepsilon=\inf \left\{\left\{\varepsilon_{i}\right\}, \varepsilon^{\prime},\left\{\theta_{k}\right\}\right\}$, nous choisissons un point $a^{\prime} \in B_{a}(\varepsilon)$ n'appartenant ni à $A_{2}$ ni à aucun des plans des triangles non dégénérés $a_{i} a_{i+1} a_{i+2}$, ni à aucune droite définie par un triangle dégénéré $a_{i} a_{i+1} a_{i+2}$. Cela implique $\left(a^{\prime} a_{i} a_{i+1}\right) \cap\left(a^{\prime} a_{i+1} a_{i+2}\right)=a^{\prime} a_{i+1}(i=1,2, \cdots, m)$.

On substitue maintenant aux faces $a a_{i} a_{i+1}$ les triangles $a^{\prime} a_{i} a_{i+1}$, ce qui remplace $A_{1}$ par une nouvelle surface simpliciale, et l'on continue de la sorte jusqu'à ce qu'on obtienne une surface simpliciale, notée encore $A_{1}$, dont aucun sommet ne coïncide avec un sommet de $A_{2}$. Si un sommet de $A_{1}$ est intérieur à une arête ou à une face de $A_{2}$, on peut appliquer la même construction, qui, répétée un nombre fini de fois, donne une surface simpliciale, notée toujours $A_{1}$, dont aucun sommet n'appartient à $A_{2}$.

(II) Supposons qu'un sommet $b$ de $A_{2}$ soit intérieur à une arête $a a_{1}$ de $A_{1}$. Comme $b \notin \partial A_{1}$, on peut supposer $a \notin \partial A_{1}$. Considérant le disque $\left(a a_{1} a_{2}\right) \cup \cdots \cup\left(a a_{m} a_{1}\right)$ des faces de $A_{1}$ autour de $a$, on détermine $\varepsilon>0$ tel que, pour tout $a^{\prime} \in B_{a}(\varepsilon)$, les conditions suivantes soient remplies: (1) Le compact $\left(a^{\prime} a_{1} a_{2}\right) \cup \cdots \cup\left(a^{\prime} a_{m} a_{1}\right)$ n'a aucun point commun avec le complémentaire du disque fermé $\left(a a_{1} a_{2}\right) \cup \cdots \cup\left(a a_{m} a_{1}\right)$ dans $A_{1}$. 
Tous les triangles $a^{\prime} a_{i} a_{i+1}$ sont non dégénérés et l'on a $\left(a^{\prime} a_{i} a_{i+1}\right) \cap$ $\left(a^{\prime} a_{j} a_{j+1}\right)=a^{\prime}$ toutes les fois où $\left(a_{i} a_{i+1}\right) \cap\left(a_{j} a_{j+1}\right)=\varnothing$. (3) Si un sommet $b^{\prime} \neq b$ de $A_{2}$ n'appartient pas à $\left(a a_{1} a_{2}\right) \cup \cdots \cup\left(a a_{m} a_{1}\right)$, il n'appartient pas non plus à $\left(a^{\prime} a_{1} a_{2}\right) \cup \cdots \cup\left(a^{\prime} a_{m} a_{1}\right)$.

Soit $a^{\prime} \in B_{a}(\varepsilon)$ un point n'appartenant ni à $A_{2}$, ni à la droite définie par $a a_{1}$, ni à aucun des plans des triangles non dégénérés $a_{i} a_{i+1} a_{i+2}$ ni à aucune des droites des triangles dégénérés $a_{i} a_{i+1} a_{i+2}$. On remplace les faces $a a_{i} a_{i+1}$ par les triangles $a^{\prime} a_{i} a_{i+1}(i=1,2, \cdots, m)$ et l'on répète ensuite la même construction autant de fois qu'il est nécessaire.

Si un sommet $b$ de $A_{2}$ est encore intérieur à une face $a a_{1} a_{2}$ de $A_{1}$, on peut supposer $a \notin \partial A_{1}$ et procéder de la même manière. On obtient finalement une surface simpliciale $A_{1}$ telle qu'aucun de ses sommets n'appartienne à $A_{2}$ et qu'aucun sommet de $A_{2}$ n'appartienne à $A_{1}$.

(III) Soit maintenant une face $a a_{1} a_{2}$ de $A_{1}$ contenant un segment d'une arête $b b_{1}$ de $A_{2}$. On peut supposer $a \notin \partial A_{1}$ et considérer le disque $\left(a a_{1} a_{2}\right) \cup$ $\cdots \cup\left(a a_{m} a_{1}\right)$ des faces de $A_{1}$ autour de $a$. Soit $\varepsilon>0$ tel que, pour tout $a^{\prime} \in B_{a}(\varepsilon)$, les conditions ci-dessous soient satisfaites: (1) Le compact $\left(a^{\prime} a_{1} a_{2}\right) \cup \cdots \cup\left(a^{\prime} a_{m} a_{1}\right)$ n'a aucun point commun avec le complémentaire de $\left(a a_{1} a_{2}\right) \cup \cdots \cup\left(a a_{m} a_{1}\right)$ dans $A_{1}$. (2) Tous les triangles $a^{\prime} a_{i} a_{i+1}$ sont non dégénérés et l'on a $\left(a^{\prime} a_{i} a_{i+1}\right) \cap\left(a^{\prime} a_{j} a_{j+1}\right)=a^{\prime}$ lorsque $\left(a_{i} a_{i+1}\right) \cap\left(a_{j} a_{j+1}\right)=\varnothing$. (3) Il n'existe pas de sommets de $A_{2}$ sur $\left(a^{\prime} a_{1} a_{2}\right) \cup \cdots \cup\left(a^{\prime} a_{m} a_{1}\right)$. (4) $\mathrm{Si}$ une arête de $A_{2}$ ne contient pas un segment appartenant à une face $a a_{i} a_{i+1}$, elle ne possède pas non plus un segment appartenant à $a^{\prime} a_{i} a_{i+1}$ $(i=1,2, \cdots, m)$.

On choisit un point $a^{\prime} \in B_{a}(\varepsilon)$ n'appartenant ni à $A_{2}$ ni au plan de $a a_{1} a_{2}$, ni à aucun des plans des triangles non dégénérés $a_{i} a_{i+1} a_{i+2}$, ni à aucune des droites des triangles dégénérés $a_{i} a_{i+1} a_{i+2}$, et on remplace les faces $a a_{i} a_{i+1}$ de $A_{1}$ par les triangles $a^{\prime} a_{i} a_{i+1} \quad(i=1,2, \cdots, m)$. Cette construction, répétée successivement, conduit à une surface simpliciale $A_{1}$ dont aucune face ne contient des segments d'arêtes de $A_{2}$.

(IV) Soit maintenant $b b_{1} b_{2}$ une face de $A_{2}$ contenant un segment d'une arête $a a_{1}$ de $A_{1}$. Supposant $a \notin \partial A_{1}$, ce qui est loisible, et considérant le disque $\left(a a_{1} a_{2}\right) \cup \cdots \cup\left(a a_{m} a_{1}\right)$ des faces de $A_{1}$ autour de $a$, on détermine un réel $\varepsilon>0$ tel que, pour tout $a^{\prime} \in B_{a}(\varepsilon)$, les conditions suivantes soient satisfaites: (1) Le compact $\left(a^{\prime} a_{1} a_{2}\right) \cup \cdots \cup\left(a^{\prime} a_{m} a_{1}\right)$ n'a aucun point commun avec le complémentaire de $\left(a a_{1} a_{2}\right) \cup \cdots \cup\left(a a_{m} a_{1}\right)$ dans $A_{1}$. (2) Les triangles $a^{\prime} a_{1} a_{2}, \cdots, a^{\prime} a_{m} a_{1}$ sont non dégénérés et l'on a $\left(a^{\prime} a_{i} a_{i+1}\right) \cap\left(a^{\prime} a_{j} a_{j+1}\right)=a^{\prime}$ toutes les fois où $\left(a_{i} a_{i+1}\right) \cap\left(a_{j} a_{j+1}\right)=\varnothing$. (3) Il n'existe pas des sommets de $A_{2}$ sur $\left(a^{\prime} a_{1} a_{2}\right) \cup \cdots \cup\left(a^{\prime} a_{m} a_{1}\right)$. (4) Aucun des triangles $a^{\prime} a_{1} a_{2}, \cdots, a^{\prime} a_{m} a_{1}$ ne contient des segments d'arêtes de $A_{2}$. (5) Si une face de $A_{2}$ ne contient pas de segment appartenant à une arête 
$a a_{i}$, elle ne possède pas non plus de segment appartenant à $a^{\prime} a_{i}(i=1$, $2, \cdots, m)$.

On choisit un point $a^{\prime} \in B_{a}(\varepsilon)$ n'appartenant ni à $A_{2}$ ni au plan de $b b_{1} b_{2}$, ni à aucun des plans des triangles non dégénérés $a_{i} a_{i+1} a_{i+2}$, ni à aucune des droites des triangles dégénérés $a_{i} a_{i+1} a_{i+2}$, et on remplace les faces $a a_{i} a_{i+1}$ de $A_{1}$ par les triangles $a^{\prime} a_{i} a_{i+1}(i=1,2, \cdots, m)$.

Par répétition de cette opération un nombre fini de fois, on obtient une surface simpliciale $A_{1}$ telle que le couple $\left(A_{1}, A_{2}\right)$ satisfasse aux deux premières conditions de la définition de la position relative canonique.

(V) Il reste à considérer le cas où l'intérieur d'une arête $a a_{1}$ de $A_{1}$ et l'intérieur d'une arête $b b_{1}$ de $A_{2}$ ont un point commun. Supposant $a \notin \partial A_{1}$ et considérant le disque des faces $a a_{i} a_{i+1}(i=1,2, \cdots, m)$ de $A_{1}$ autour de $a$, on détermine un réel $\varepsilon>0$ tel que, pour tout $a^{\prime} \in B_{a}(\varepsilon)$, les conditions (1), (2), (3), (4) de (IV) et en outre les deux conditions ci-après soient vérifiées. (5) Aucune des arêtes $a^{\prime} a_{1}, \cdots, a^{\prime} a_{m}$ ne contient des segments appartenant à des faces de $A_{2}$. (6) Si l'intérieur d'une arête de $A_{2}$ ne rencontre pas l'intérieur d'une arête $a a_{i}$, il ne rencontre pas non plus l'intérieur de $a^{\prime} a_{i}(i=1,2, \cdots, m)$.

On choisit un point $a^{\prime} \in B_{a}(\varepsilon)$ n'appartenant ni à $A_{2}$, ni au plan des $a a_{1}$ et $b b_{1}$, ni à aucun des plans des triangles non dégénérés $a_{i} a_{i+1} a_{i+2}$, ni à aucune des droites des triangles dégénérés $a_{i} a_{i+1} a_{i+2}$, et on remplace les faces $a a_{1} a_{2}, \cdots, a a_{m} a_{1}$ de $A_{1}$ par les triangles $a^{\prime} a_{1} a_{2}, \cdots, a^{\prime} a_{m} a_{1}$. Ce genre de déformation, répété un nombre fini de fois, conduit à une surface simpliciale ayant même bord que la surface de départ $A_{1}$ et se trouvant en position canonique relativement à $A_{2}$.

La Proposition 2.3 est ainsi établie.

Si l'on remarque que tous les homéomorphismes effectués déplacent les points de $A_{1}$ à des distances aussi petites que l'on veut, on peut énoncer la

Proposition 2.4. Soient $A_{1}$ et $A_{2}$ deux surfaces simpliciales de $R^{3}$ telles que $A_{1} \cap A_{2} \neq \varnothing$. Si $A_{2}$ est sans bord et si $A_{1} \cap A_{2}$ ne rencontre pas $\partial A_{1}$, on peut, quel que soit $\varepsilon>0$, déterminer un isomorphisme simplicial $\xi$ de $A_{1}$ sur une surface simpliciale $A_{1}^{\prime}$ de $R^{3}$ de façon que (1) $|\xi(x)-x|<\varepsilon$ pour tout $x \in A_{1}$, (2) $\xi(x)=x$ pour tout $x \in \partial A_{1}$, (3) $A_{1}^{\prime}=\xi\left(A_{1}\right)$ et $A_{2}$ se trouvent en position relative canonique.

Lorsque $A_{2}$ possède aussi un bord, le résultat reste évidemment valable toutes les fois où $A_{1} \cap A_{2}$ ne rencontre pas $\partial A_{1} \cup \partial A_{2}$. En cas contraire, on peut construire l'homéomorphisme $\xi$ de façon que la première et la troisième des conditions ci-dessus soient satisfaites, mais pas la deuxième.

3. Généralisation du théorème d'Antoine. Soient $C$ un cylindre ordinaire, $b$ et $b^{\prime}$ ses bases, $l$ son axe. Un polyèdre homéomorphe à ce 
cylindre sera noté $C\left(b, b^{\prime} ; l\right)$ toutes les fois où il sera muni d'une triangulation dans laquelle les images des $b$ et $b^{\prime}$ sont des disques simpliciaux et l'image de $l$ est une réunion de 1 -simplexes.

Un polyèdre triangulé de $R^{3}$ sera dit un tore solide simplicial d'âme $\Lambda$, s'il est un tore solide formé d'une réunion finie de polyèdres $C_{i}\left(b_{i}, b_{i}^{\prime} ; l_{i}\right)$ $(i=1,2, \cdots, n)$ tels que

(1) $C_{i}\left(b_{i}, b_{i}^{\prime} ; l_{i}\right) \cap C_{i+1}\left(b_{i+1}, b_{i+1}^{\prime} ; l_{i+1}\right)=b_{i}^{\prime}=b_{i+1}$ pour $i=1,2, \cdots, n-1$,

(2) $C_{n}\left(b_{n}, b_{n}^{\prime} ; l_{n}\right) \cap C_{1}\left(b_{1}, b_{1}^{\prime} ; l_{1}\right)=b_{n}^{\prime}=b_{1}$,

(3) $C_{i}\left(b_{i}, b_{i}^{\prime} ; l_{i}\right) \cap C_{j}\left(b_{j}, b_{j}^{\prime} ; l_{j}\right)=\varnothing$ si $i$ et $j$ ne sont pas consécutifs $\bmod n$,

(4) $\Lambda=\bigcup_{1}^{n} l_{i}$ soit un cycle polygonal.

L'image de $\left\{\left(x_{1}, x_{2}\right) \in R^{2} \mid 1 \leqq x_{1}^{2}+x_{2}^{2} \leqq 2\right\}$ dans $R^{3}$ par une injection continue sera appelée un fermé annulaire topologique de $R^{3}$. Un fermé annulaire topologique sera appelé un fermé annulaire simplicial s'il est une surface simpliciale.

Proposition 3.1. Considérons dans $R^{3}$ deux cycles polygonaux $\Lambda$ et $L$ simplement enlacés. Soit $A$ un fermé annulaire simplicial de $R^{3}$ limité par les cycles polygonaux $\Gamma_{1}$ et $\Gamma_{2}$ et tel que $A \cap \Lambda=\varnothing$ et $A \cap L=\varnothing$. Soient $\mu$ et $\nu$, considérés non négatifs, les nombres d'enlacement de $\Gamma_{1}$ avec $\Lambda$ et $L$ respectivement. S'il existe un tore solide simplicial d'âme $\Lambda$ contenant $\Gamma_{1}$ à son intérieur et laissant $\Gamma_{2}$ et $L$ à son extérieur, et si $\mu>1$ et $v>1$, ces deux entiers sont premiers entre eux.

Démonstration. Soit $F$ la frontière du tore solide. D'après la Proposition 2.4, il existe un fermé annulaire simplicial $A^{\prime}$ en position canonique relativement à $F$, limité par $\Gamma_{1}$ et $\Gamma_{2}$, et tel que $A^{\prime} \cap \Lambda=\varnothing$ et $A^{\prime} \cap L=\varnothing$. D'après la Proposition 2.2, $A^{\prime} \cap F$ est formé d'un nombre fini de cycles polygonaux deux à deux disjoints. Soient $z_{1}, z_{2}, \cdots, z_{n}$ les cycles polygonaux de $A^{\prime} \cap F$ qui sont homologues à zéro dans $A^{\prime}$ et qui jouissent de la propriété suivante: Pour tout $z_{i}(i=1,2, \cdots, n)$, il n'existe aucun autre cycle polygonal de $A^{\prime} \cap F$ homologue à zéro dans $A^{\prime}$ et limitant (dans $A^{\prime}$ ) un disque contenant $z_{i}$. Nous traçons dans $A^{\prime}$ des cycles polygonaux $z_{1}^{\prime}, z_{2}^{\prime}, \cdots, z_{n}^{\prime}$ deux à deux disjoints, ne rencontrant ni $A^{\prime} \cap F$ ni $\Gamma_{1} \cup \Gamma_{2}$ et tels que, pour tout $i \in\{1,2, \cdots, n\}, z_{i}^{\prime}$ limite dans $A^{\prime}$ un disque $D_{i}^{\prime}$ contenant à son intérieur le seul cycle $z_{i}$ parmi les cycles $z_{1}, z_{2}, \cdots, z_{n}$. Supprimant de $A^{\prime}$ les intérieurs des $D_{i}^{\prime}$ dans $A^{\prime}$, on obtient un fermé connexe $A^{\prime \prime}$ qui ne contient aucun cycle polygonal de $A^{\prime} \cap F$ homologue à zéro dans $A^{\prime}$. Comme $\Gamma_{1}$ et $\Gamma_{2}$ font partie de $\partial A^{\prime \prime}$, l'intersection $A^{\prime \prime} \cap F$ n'est pas vide. Elle ne comprend d'ailleurs que des cycles de $A^{\prime} \cap F$. Il existe donc dans $A^{\prime} \cap F$ un cycle polygonal $y$ qui n'est pas homologue à zéro dans $A^{\prime}$. Mais alors $y \sim \Gamma_{1}$ dans $A^{\prime}$, donc aussi dans $R^{3}-\Lambda$ et dans 
$R^{3}-L$, et cela prouve que les nombres d'enlacement de $y$ avec $\Lambda$ et $L$ sont respectivement $\mu$ et $\nu$. Comme $y \subset F$, le théorème d'Antoine est applicable. Les entiers $\mu$ et $\nu$ sont bien premiers entre eux.

Proposition 3.2. Soient, dans $R^{3}, \Lambda$ et $L$ deux cycles polygonaux simplement enlacés. Soit $A$ un fermé annulaire topologique de $R^{3}$ limité par les courbes de Jordan $\Gamma_{1}$ et $\Gamma_{2}$ et tel que $A \cap \Lambda=\varnothing$ et $A \cap L=\varnothing$. Soient $\mu$ et $v$, considérés non négatifs, les nombres d'enlacement de $\Gamma_{1}$ avec $\Lambda$ et $L$ respectivement. S'il existe un tore solide simplicial d'âme $\Lambda$ contenant $\Gamma_{1}$ à son intérieur et laissant $\Gamma_{2}$ et $L$ à son extérieur, et si $\mu>1$ et $\nu>1$, ces deux entiers sont premiers entre eux.

DÉmonstration. Soient $\varepsilon_{1}$ la distance entre $A$ et $\Lambda, \varepsilon_{2}$ la distance entre $A$ et $L, \varepsilon_{3}$ la distance entre $\Gamma_{1}$ et la frontière $F$ du tore solide, $\varepsilon_{4}$ la distance entre $\Gamma_{2}$ et $F$. Soit $\varepsilon=\inf \left\{\varepsilon_{1} / 2, \varepsilon_{2} / 2, \varepsilon_{3} / 2, \varepsilon_{4} / 2\right\}$. D'après le théorème d'approximation [2] de Bing, il existe un homéomorphisme $\xi$ appliquant $A$ sur un fermé annulaire simplicial $A^{\prime}$ et tel que $|\xi(x)-x|<\varepsilon$ pour tout $x \in A$. $A^{\prime}$ est limité par deux cycles polygonaux $\Gamma_{1}^{\prime}$ et $\Gamma_{2}^{\prime}$ tels que $\Gamma_{1} \sim \Gamma_{1}^{\prime}$ et $\Gamma_{2} \sim \Gamma_{2}^{\prime}$ dans $R^{3}-(\Lambda \cup L)$. Il s'ensuit que les nombres d'enlacement de $\Gamma_{1}^{\prime}$ (et de $\Gamma_{2}^{\prime}$ ) avec $\Lambda$ et $L$ sont respectivement $\mu$ et $v$. Comme $\Gamma_{1}^{\prime}$ et $\Gamma_{2}^{\prime}$ se trouvent respectivement à l'intérieur et à l'extérieur du tore solide, la Proposition 3.1 donne le résultat.

Soit $\varepsilon$ un réel positif. Etant donné une courbe de Jordan $\Gamma$ dans $R^{3}$, on peut déterminer un cycle polygonal $\Lambda \subset R^{3}$ et un homéomorphisme $\xi$ : $\Gamma \rightarrow \Lambda$ tel que $|\xi(x)-x|<\varepsilon$ pour tout $x \in \Gamma$. Nous appellerons $\Lambda$ une approximation polygonale canonique de $\Gamma$.

Proposition 3.3. Soit $f:[0,1] \times S \rightarrow R^{3}$ l'application déjà définie $(\S 1)$. Soient $L$ un cycle polygonal simplement enlacé avec $\Gamma_{0}$ et $\left.\left.t_{2} \in\right] 0,1\right]$ une valeur telle que $\operatorname{L} \cap\left(\left[0, t_{2}\right] \times S\right)=\varnothing$. Supposons qu'il existe une approximation polygonale canonique $\Lambda$ de $\Gamma_{0}$ donnant lieu aux propriétés suivantes:

(1) Il existe un tore solide simplicial $T$ d'âme $\Lambda$ contenant $\Gamma_{0}$ à son intérieur et laissant à son extérieur la courbe $\Gamma_{t_{2}}$ et le cycle $L$.

(2) Il existe une valeur positive $t_{1}<t_{2}$ telle que $\Gamma_{t_{1}}$ se trouve à l'intérieur de $T$ et que le fermé annulaire topologique $f\left(\left[t_{1}, t_{2}\right] \times S\right)$ n'ait aucun point commun avec $\Lambda$.

Dans ces conditions, si $\mu>1$ et $\nu>1$, ces deux entiers sont premiers entre eux.

En effet, d'après la Proposition 2.1, le nombre d'enlacement des courbes $\Gamma_{t}, 0<t \leqq t_{2}$, avec $L$ est $v$. Il en est ainsi en particulier des courbes $\Gamma_{t_{1}}$ te $\Gamma_{t_{2}}$, qui constituent le bord du fermé annulaire topologique $f\left(\left[t_{1}, t_{2}\right] \times S\right)$. Le résultat est donc une conséquence immédiate de la Proposition 3.2. 
COROLlaire 3.3. fétant toujours l'application définie dans $§ 1$, si $\Gamma_{0}$ est un cycle polygonal et si $\mu>1$ et $\nu>1$, ces deux entiers sont premiers entre eux.

DÉmonstration. Soient $L$ un cycle polygonal simplement enlacé avec $\Gamma_{0}$ et $\left.\left.t_{2} \in\right] 0,1\right]$ une valeur pour laquelle $L \cap f\left(\left[0, t_{2}\right] \times S\right)=\varnothing$. Soit $T$ un tore solide simplicial d'âme $\Gamma_{0}$ laissant à son extérieur la courbe $\Gamma_{t_{2}}$ et le cycle $L$. Il suffit alors de choisir une valeur positive $t_{1}<t_{2}$ pour laquelle la courbe $\Gamma_{t_{1}}$ soit à l'intérieur de $T$.

\section{RÉFÉRENCES}

1. L. Antoine, Sur l'homéomorphie de deux figures et de leurs voisinages, J. Math. Pures Appl. (8) 4 (1921), 221-325.

2. R. H. Bing, Approximating surfaces with polyhedral ones, Ann. of Math. (2) 65 (1957), 456-483. MR 19; 300.

99, rue de Sèvres, 92 Boulogne, France 